\title{
Chapter 29 \\ Possible Experiments with Heavy Ions at the PS/SPS: CERN SPC 1982
}

\author{
Johann Rafelski
}

\begin{abstract}
I present the heavy ion program development at CERN, reproducing much of the pivotal discussion at the 123th meeting of the CERN Scientific Policy Committee (SPC), Geneva-21 and 22 June 1982, based on the Draft Minutes of the meeting (CERN/SPC/0490/Draft, 1982) and related clarifications as marked.
\end{abstract}

\subsection{The Participants}

The CERN Scientific Policy Committee meeting in June 1982 brought together a large invited group that included the international particle physics leadership.

Chairman: Prof. V.L. Telegdi Members:

Prof. I. Bergström Prof. N. Cabibbo Prof. P. Falk-vairant

Prof. S.L. Glashow Prof. E. Lohrmann Prof. L.B. Okun

Prof. D.H. Perkins Prof. Abdus Salam Prof. G. Salvini

Dr. G.H. Stafford Prof. W. Thirring Prof. K. Tittel

Dr. R. Turlay.

Ex Officio Members:

Prof. G. Bellettini, Chairman-ISR Committee

Prof. P.G. Hansen, Chairman-PS/SC Committee

Prof. J. Lefrançois, Chairman-SPS Experiments Committee

Dr. J.H. Mulvey Invited in his capacity as Chairman of ECFA

Also present:

Prof. K.O. Nielsen-Chairman of the Finance Committee

Prof. J.C. Kluyver

Prof. J. Lemonne

Editor of the SPC Protocol

J. Rafelski $(\bowtie)$

Department of Physics, The University of Arizona, Tucson, AZ 85721, USA

(C) The Author(s) 2016

J. Rafelski (ed.), Melting Hadrons, Boiling Quarks - From Hagedorn Temperature

to Ultra-Relativistic Heavy-Ion Collisions at CERN,

DOI 10.1007/978-3-319-17545-4_29 
Prof. P. Olesen

Prof. A.C. Pappas

Former Members Invited:

Prof. E. Amaldi

Prof. M. Conversi

Prof. A.G. Ekspong

Prof. B. Hahn

Prof. W. Jentschke

Prof. A. Lehmann

Prof. L. Leprince-Ringuet

Prof. P.T. Matthews

Prof. W. Paul

Prof. F. Perrin

Prof. A. Rousset

Prof. S.A. Wouthuysen

CERN Officials: Prof. H. Schopper-CERN Director-General

Dr. G. Brianti-Technical Director

Dr. E. Gabathuler-Research Director

Prof. R. Klapisch-Research Director

Prof. E. Picasso-Director and LEP Project Leader

Invited: Dr. M. Jacob for the "Heavy Ion Collisions" item of the agenda

\subsection{On Formation of QGP in Heavy Ion Collisions}

Maurice Jacob begins his presentation at 11:20, 22 June, 1982.

"Heavy ion collisions offer the possibility to reach very high densities and very high temperatures over extended domains, many times larger than the size of a single hadron. The energy densities considered are of the order of $0.5-1.5 \mathrm{GeV} / \mathrm{fm}^{3}$ and the relevant temperatures are in the $200 \mathrm{MeV}$ range. The great interest of reaching such conditions originates from recent developments in Quantum Chromodynamics, QCD, which make it very plausible that, while color confinement should prevail under standard circumstances, deconfinement should occur at sufficiently high density and (or) sufficiently high temperature. Under such conditions a new phase of matter, a quark-gluon plasma, is likely to exist. This phase should be viewed as due to a coalescence, or perhaps a percolation, of hadrons into larger entities and not as an actual separation of free quarks!...

"Over an extended volume where the required density or temperature conditions would prevail, one expects that the properties of the physical vacuum would be modified. While the normal vacuum excludes the gluon field, the color-equivalent of the dielectric constant being zero (or practically zero), one would get a new vacuum state where quarks and gluons could propagate while interacting perturbatively.

"The equivalent of the dielectric constant would now be unity. The required conditions may be reached at high enough densities, hadrons being squeezed into one another, or at high enough temperature, the calculation of the partition function no longer favoring confining configurations whereby a color flux tube of fixed cross section extends between two color sources. The temperature at which the phase transition is expected to occur depends on the density, or on the quark chemical potential. One may thus separate two phases, a hadron phase and a quark gluon plasma, on a density-temperature diagram....

"The presence of a phase transition could long be expected from phenomenological models with an exponentially increasing hadron spectrum. The limiting Hagedorn temperature, obtained as the specific heat of the hadron gas diverges, can 
be interpreted as a critical temperature beyond which the relevant description should be in terms of a quark-gluon plasma reaching eventually a Stephan-Boltzmann behavior. The actual presence of a phase transition finds however its strongest present support in lattice gauge calculations....

"Granting the fact that phase transition(s) exist(s), the next question is to assess whether or not the required conditions could be met in heavy ion collisions with center-of-mass energy in excess of $10 \mathrm{GeV} /$ nucleon. At present there also appears to be a consensus that this is the case....

"The expected mean energy density is of the order of $2 \mathrm{GeV} / \mathrm{fm}^{3}$ for the (most favorable) case of head on U-U collisions and still of the order of $1.2 \mathrm{GeV} / \mathrm{fm}^{3}$ for $\mathrm{Fe}-\mathrm{Fe}$ collisions. This applies to the fragmentation region, considering the energy trapped in what remains of the projectile or target nucleus just after the collision....

"Granting the fact that a thermalized quark gluon plasma is formed during the collision, it will very rapidly destroy itself through instabilities, expansion and cooling. One should then watch for specific signals which could be associated with its transient (but most interesting) presence. ...

"Several signals have attracted particular attention.

1. One of them is provided by the prompt photon or lepton pairs radiated (a volume effect!) by the thermalized plasma, ...

2. Another interesting signal may be provided by strange particles originating in relatively large number from the plasma, once it has reached chemical equilibrium.

3. There may also be more violent effects, with abnormal density fluctuations in the overall energy flow associated with secondaries.

4. Size and lifetime could be determined through pion/photon interferometry since each violent event with head on collision could produce pions in the thousands!

\subsection{Experimental Opportunities to Study QGP}

At the recent Bielefeld workshop.

“... six working groups studied experimental questions from the point of view of physics goals and their technical realization...

1. The group convened by S. Nagamiya and H. Specht studied measurement of inclusive particle distributions. It became clear that the desired measurements were single particle spectra, not necessarily truly inclusive, but with various triggers to select central collisions and those with large multiplicity or energy deposit in the target.

From the physics side, it was established that a good way to investigate the effects in the quark-gluon plasma such as the suppression of $u$-quarks and the chemical equilibrium of $s$-quarks, should be to measure distributions of strange particles, mesons and especially strange and multiply strange baryons and anti- 
baryons. ... It was concluded that the presence of very high multiplicities does not present a major obstacle to these experiments.

... It is certainly reasonable to expect to find many strange quarks (and some charmed quarks) lodged in the fragments of the projectile. The large Lorentz factor will then allow the use of beams similar to the existing hyperon beams to provide momentum analyzed, mass and charge identified hypernuclei. There should also be usable numbers of multiply strange hypernuclei. This is a radically new approach to the study of these particles and should give rise to a major step forward.

2. Experiments on correlations among a few particles were considered by the group convened by I. Otterlund and H. Boggild. The idea here was to handle the high multiplicities by using spectrometers with a solid angle just large enough to cover the angles between two particles in the range of interest, but small enough so that the number of particles to be measured is still close to that commonly encountered....

The topic of identical particle intensity interferometry by study of few particle correlations was given special attention. This technique has already proved its worth in nuclear collisions and is expected to be a major tool in high energy nucleus-nucleus interactions. It is used to measure the size and shape of the interaction volume,....

This apparatus also seems suited for studies of V0's. The group also designed a special spectrometer to study photon correlations. This group devoted a substantial effort to the study of various triggers to select central or peripheral events, including measurement of the forward particles and a "plastic ball" type of detector covering most of the solid angle of target fragmentation.

3. G. London and K. Nakai convened a group working on the production of leptons and photons. They established that several distinct kinematic regions seemed to be of interest. For intermediate and high mass muon pair production in the projectile fragment region, they showed that an experiment using the NA3 apparatus at the SPS with small modification could be very effective....

4. A combined group convened by C. Fabjan, H. Gutbrod, A. Sandoval and A. Wagner studied colorimetric techniques and tracking devices in large solid angle detectors. The beauty of energy flow measurements with calorimeters is well recognized, but this group took the attitude that there would be powerful arguments for an apparatus which could make nearly complete measurements on an event by event basis and set out to investigate if it is technically feasible using methods presently available....

5. Another working group convened by M. Faessler and S. Frankel studied the case of deuteron and alpha particle beams...

6. A group convened by R. DeVries and H.G. Fischer worked on the subject of peripheral interactions. A major part of their time was devoted to the study of the experiments at Berkeley giving particles with very short interaction lengths .... 
"The large variety of experiments devised by the working group indicates a need to run several experiments at one time. The intensity requirements of the experiments are such that this should be possible....

"Concluding this rapid survey of the physics of heavy ion collisions, one may say that there is practically no doubt that a phase transition exists, even if the exact form which it takes is not yet precisely known. There is also practically no doubt that the energy density to be achieved in heavy ion collisions, with incident ion beams in the $200 \mathrm{GeV} /$ nucleon energy range, should reach the critical value....

\subsection{Discussion on Relativistic Heavy Ion Collisions}

The chairman, Prof. H. Schopper, thanked Maurice Jacob for his presentation, and opened the discussion.

Replying to a question from Prof. P.T. Matthews, Maurice Jacob said that the fundamental purpose of heavy-ion collision experiments was to study matter at very high quark densities. It was thought that when such densities were created, a new phase of matter appeared which would signal its existence by an anomalous production of photons, lepton-pairs or strange particles. Heavy-ion collision experiments would therefore be designed to investigate this anomalous production. It was possible that even more peculiar effects could be associated with high quark densities, but he had concentrated on the conservative ones which one could expect to see from a blob of natter at a temperature of the order of $200 \mathrm{MeV}$. At this energy the blob would radiate photons and its gluons would transform favorably into pairs. Experiments would therefore be designed to observe and search for large fluctuations in specific parameters. It was expected that the production mechanism would show up clearly in heavy-ion collisions, whereas there was no evidence for, and little hope to reach, such energy densities over an extended domain in protonnucleus collisions.

Prof. P.G. Hansen, PS/SC Committee Chair added that one of the essential aspects of any experiment would be to study the question at different energies to determine how much energy was required for the formation of quark-gluon plasma, JR. In such experiments there were three essential variables: the target mass, the projectile mass and the energy. Unfortunately, the projectile mass was not available at all energy scales, and therefore, for the time being, only relatively light projectiles at very high energies could be considered. This increased the importance of repeating the experiment at different energies to ascertain whether the signature variables showed any characteristic change which could indicate the existence of the phase transition. It was in this context that the discussion centered on the use of the PS as a step on the way to $200 \mathrm{GeV}$ per nucleon.

Prof. D.H. Perkins observed that, as the atomic number of colliding ions increased, there must be a critical point where plasma effects became important, but it was difficult to see how this point could be determined owing to the large energy density fluctuations. 
Maurice Jacob, replying, said that theoretical efforts were currently being concentrated on obtaining mean values of energy densities which could be expected in a collision of this kind. There were bound to be large fluctuations, and while there was some information about fluctuations in $p p, p$-nucleus and $\alpha \alpha$ collisions, as yet there was no information on how significant these fluctuations could be in the case of heavy-ion collisions. Information about such fluctuations was a very important reason for experimentation and indeed, density fluctuations towards large values were probably those needed for the phase transition to take place.

The chairman, Prof. H. Schopper pointed out that, with regard to the question of particle signature, it should first be established in what rare fraction of cases, using the standard theory, such phenomena would take place. It ought to be possible, for example, to predict the probability that 1,000 pions would be produced at the reference energies without invoking such phenomena as the phase transition predicted by QCD.

Maurice Jacob replying, said that the standard theories would predict that the mean multiplicity would rise from between $A^{2 / 3}$ to $A^{4 / 3}$ according to the model. The observation of very large multiplicities, showing that a large amount of energy could be found in excitation energy, could be considered as a necessary condition for a phase transition.

Regarding fluctuations away from the mean, information was available in the case of proton/proton collisions where the fluctuations had been very well characterized in terms of the KNO distribution up to a certain value. Thereafter, practically nothing was known about collisions with extremely high multiplicities because they were so difficult to study. The results of the NA5 experiment had emphasized this point, showing, for example, that, when looking for large amounts of transverse energy, the production of a very large number of particles with medium $p_{\perp}$ might prove to be a more frequent phenomenon than the production of a few particles with large $p_{\perp}$ associated with jets.

Replying to questions from Prof. D.H. Perkins and the chairman, Prof. H. Schopper, Maurice Jacob said that collisions with a projectile with a large atomic number were required because the amount of deposited energy was proportional to the number of nucleons in the incident nucleus. Estimates suggested that, in the most optimistic case of head-on uranium/uranium collisions, energy densities of the order of $2 \mathrm{GeV} / \mathrm{fm}^{3}$ would be obtained, whereas in the case of carbon/uranium collisions, this figure would fall to $1 \mathrm{GeV} / \mathrm{fm}^{3}$.

Prof. G. Bellettini, ISR Committee Chairman, I observed that, not withstanding the obvious advantages of heavy-ion collisions, it would be interesting to ascertain experimentally whether anomalous phenomena could be observed with $p p$ and/or $\alpha \alpha$ collisions.

Replying to a question from Prof. E. Amaldi, Maurice Jacob said that, with regard to the question of the time necessary for the plasma to achieve equilibrium, it was expected that there was a chance that some thermalization would take place at the level of the quarks and the gluons present in the plasma, many collisions having time to take place. 
Replying to Prof. N. Cabibbo, Maurice Jacob said that the Helsinki group in particular had estimated lepton pair production in detail. In accordance with the standard thermodynamic formulae, the number of photons produced in the plasma depended upon the charged-particle density and the temperature. Since this would essentially be a volume effect, the larger the volume of the plasma the greater would be the increase in photon production with respect to pions. Consequently, the volume of the plasma was an important parameter to determine.

In reply to a question from Prof. A.G. Ekspong, about the anomalous effect termed the anomalon, Maurice Jacob said that its interpretation as the decay of a hyperfragment of a strange particle had now been rejected. Research at the Bevalac at Berkeley had revealed that, when observed close to production, some fragments seemed to have very large cross-sections for a given ionizing power, ... . Purely experimental problems should, however, not be underestimated. ${ }^{1}$

Replying to Dr R. Turlay, Maurice Jacob said that what was particularly new in this type of physics was the expected production of lepton pairs at large $x$. If a new state of matter existed, one could foresee that it would radiate lepton pairs and that their momentum would correspond essentially to the global motion of the blob of matter. Any experiment would therefore concentrate on looking for lepton pairs at large $x$ with a thermal-type mass distribution as opposed to the $1 / \mathrm{m}^{4}$ distribution for the $d \sigma / d m^{2}$ distribution, associated with the Drell-Yan theory with a concentration at low $x$. One would expect to see a very sharp fall-off of the lepton-pair mass spectrum as compared to the Drell-Yan spectrum.

Replying to a question from Prof. J. Lefrançois, SPS Experiments Chairman, Maurice Jacob said that at $1 \mathrm{GeV} / \mathrm{fm}^{3}$ the temperature of the plasma would be too low for significant production of charm and beauty particles.

In reply to a question from Prof. N. Cabibbo, Maurice Jacob said that the great merit of the QCD calculation using the lattice over the Hagedorn model was that it made direct exploration of the system possible over and beyond the phase transition, whereas the phenomenological model had been based on a separate study of the two phases. The two approaches were, however, complementary, in many respects. What the experimenters wished to do with heavy-ion collision experiments was to ascertain whether matter existed in a different form beyond the hadron gas.

The chairman, Prof. H. Schopper, in conclusion, said it was clear that any discussion of heavy-ion collision experiments raised as many questions as it attempted to resolve. However, before very long the Scientific Policy Committee would have to address itself to the question of heavy-ion collision experiments in a more formal way. When the proceedings of the Bielefeld Workshop had been

\footnotetext{
${ }^{1}$ An analysis offered by I. Otterlund a year later (lecture at the Sixth High Energy Heavy Ion Study, Berkeley, 28 June-1 July 1983) has shown that the bias of human eye-based-analysis was the source of the shortened reaction path observed; see also S.B. Beri et al. [Banaras-ChandigarhJaipur-Jammu-Lund Collaboration], "A Search for Anomalous Fragments in $1.8 \mathrm{~A} \mathrm{Gev}{ }^{40} \mathrm{Ar}$ Reactions in Nuclear Emulsions," Phys. Rev. Lett. 54 (1985) 771. JR.
} 
published, the Committee would be in a position to brief itself more thoroughly in order to formulate an appropriate recommendation.

The Committee took note of the report, and of the further explanations provided by Maurice Jacob.

The chairman, Prof. H. Schopper, said that before concluding the proceedings he wished to ask any of the former members of the Committee whether they had any comments or statements of a general nature to make.

Prof. L. Leprince-Ringuet said that, although no longer directly associated with the affairs of CERN, he nevertheless continued to follow its development with great interest. In this respect, he particularly appreciated the opportunity afforded him by this meeting of the Scientific Policy Committee to become acquainted with the latest developments in particle physics research and to hear about the progress achieved in specific projects.

In general terms, however, he was increasingly bewildered by the size and complexity of CERN's activities and of individual experiments, which could involve hundreds of physicists and whose leaders were thus no longer experimentalists in the true sense of the word but administrators. He was concerned that this preoccupation with size and a concomitantly high degree of organization could have the effect of reducing flexibility and the ability of scientists to maintain an open-minded approach to the problems with which they were concerned.

Increasingly, it seemed, experimentalists were informed in advance of the phenomena they would encounter. This elevation of the theorist to pre-eminence could have the detrimental effect of reducing the receptiveness of the experimentalist to the unexpected, weighed down as he was by the sheer volume of data to be analyzed. It should never be forgotten that most of the major discoveries made in the field of particle physics during the century had been unforeseen.

Prof. E. Amaldi said that while he did not share Leprince-Ringuet's concern that the size of experiments must necessarily limit their success, for he was certain that new discoveries would emerge before long, he doubted whether a member of a modern collaboration of, say, 250 physicists could derive as much pleasure and satisfaction from an experiment as had physicists of his own generation.

On behalf of the Committee, the chairman, Prof. H. Schopper, expressed thanks to all former members of the Scientific Policy Committee for their contributions during the meeting, and to the three members now leaving the Committee-Prof. G. Salvini, Dr. G.H. Stafford and Prof. W. Thirring-for their work.

The meeting ended at 13.15 -after $1 \mathrm{~h} 55$ min mostly if not exclusively devoted to the discussion of the future heavy ion program at CERN.

Open Access This book is distributed under the terms of the Creative Commons Attribution Noncommercial License which permits any noncommercial use, distribution, and reproduction in any medium, provided the original author(s) and sources are credited. 